\title{
On Pulsar Distance Measurements And Their Uncertainties
}

J.P.W. Verbiest

J.M. Weisberg

A. A. Chael

K.J. Lee

D. R. Lorimer

Follow this and additional works at: https://researchrepository.wvu.edu/faculty_publications

\section{Digital Commons Citation}

Verbiest, J. P. W.; Weisberg, J. M.; Chael, A. A.; Lee, K. J.; and Lorimer, D. R., "On Pulsar Distance Measurements And Their Uncertainties" (2012). Faculty Scholarship. 623.

https://researchrepository.wvu.edu/faculty_publications/623 


\title{
ON PULSAR DISTANCE MEASUREMENTS AND THEIR UNCERTAINTIES
}

\author{
J. P. W. Verbiest ${ }^{1}$, J. M. Weisberg ${ }^{2}$, A. A. Chael ${ }^{2}$, K. J. LeE ${ }^{1}$, And D. R. Lorimer ${ }^{3}$ \\ ${ }^{1}$ Max-Planck-Institut für Radioastronomie, Auf dem Hügel 69, 53121 Bonn, Germany \\ 2 Department of Physics and Astronomy, Carleton College, Northfield, MN 55057, USA \\ ${ }^{3}$ Department of Physics, West Virginia University, Morgantown, WV 26506, USA \\ Received 2012 February 14; accepted 2012 June 1; published 2012 July 24
}

\begin{abstract}
Accurate distances to pulsars can be used for a variety of studies of the Galaxy and its electron content. However, most distance measures to pulsars have been derived from the absorption (or lack thereof) of pulsar emission by Galactic H I gas, which typically implies that only upper or lower limits on the pulsar distance are available. We present a critical analysis of all measured $\mathrm{H}$ I distance limits to pulsars and other neutron stars, and translate these limits into actual distance estimates through a likelihood analysis that simultaneously corrects for statistical biases. We also apply this analysis to parallax measurements of pulsars in order to obtain accurate distance estimates and find that the parallax and H I distance measurements are biased in different ways, because of differences in the sampled populations. Parallax measurements typically underestimate a pulsar's distance because of the limited distance to which this technique works and the consequential strong effect of the Galactic pulsar distribution (i.e., the original Lutz-Kelker bias), in H I distance limits, however, the luminosity bias dominates the Lutz-Kelker effect, leading to overestimated distances because the bright pulsars on which this technique is applicable are more likely to be nearby given their brightness.
\end{abstract}

Key words: astrometry - pulsars: general

Online-only material: Supplemental data file (tar.gz)

\section{INTRODUCTION}

The rotation of pulsars, which causes their continuous emission to be observed as highly regular pulses, makes these objects highly useful probes of any dispersive phenomena in interstellar space. Combined with an accurate and precise distance, pulsar emission (specifically its dispersion and Faraday rotation) provides crucial information for modeling of the Galactic electron distribution and magnetic field.

Parallax measurements are non-trivial undertakings and only very few significant parallax measurements (Gwinn et al. 1986; Bailes et al. 1990) were made within the first two decades after pulsars were discovered. Another method to determine a pulsar's distance is based on Galactic $\mathrm{H}$ I spectra in the direction to the pulsar. This method (known as the kinematic or $\mathrm{HI}$ method) compares the H I spectrum on-pulse (when the pulsar emission is seen) and off-pulse (when the pulsar emission beam is turned away). Any observed pulsar absorption must originate in gas lying closer than the pulsar; while gas located farther than the pulsar will not exhibit absorption. The velocities of these respective $\mathrm{H}$ I regions are subsequently derived from the spectrum and translated to distances with the help of a Galactic rotation model. The distance of the furthest $\mathrm{H}$ I gas that appears in absorption then provides a lower limit $D_{\text {low }}$ on the pulsar distance, while the distance of the nearest gas that only appears in emission is interpreted as an upper limit $D_{\text {up }}$ on the pulsar distance.

Roughly two decades after the discovery of pulsars, Frail \& Weisberg (1990, henceforth FW90) collated all published pulsar distances, which at the time consisted of $50 \mathrm{H} \mathrm{I}$ distances, three parallax measurements, and 20 distances by association. Given the importance of $\mathrm{HI}$ distances, they critically investigated the various measurements and defined a set of criteria that has been used in almost all subsequent publications.

Progress in both interferometric hardware (at the Long Baseline Array in the South and the Very Long Baseline Array in the North) and in the sensitivity of pulsar timing, subsequently allowed an exponential increase in the number of measured pulsar parallaxes so that currently 57 parallaxes are measured. This led Verbiest et al. (2010, henceforth VLM10) to collate those distances and investigate the statistical bias predicted by Lutz \& Kelker (1973). The work presented by VLM10 was based on a Bayesian analysis that took into account both the Galactic distribution of pulsars (which is the actual bias first discussed by Lutz \& Kelker in 1973) and the intrinsic pulsar luminosity distribution, but they only considered parallax measurements.

In this paper, we present an update of the work done by FW90: we list all 80 published distances to pulsars and other neutron stars, based on $\mathrm{HI}$ measurements or associations with objects having $\mathrm{H}$ I distances, and evaluate them based largely on the criteria laid out by FW90. We then improve the analysis of VLM10 by deriving fully analytic solutions that replace the need for (approximate) Monte Carlo simulations. Also, the VLM10 analysis is expanded to incorporate information provided by $\mathrm{HI}$ distance limits and to provide bias-corrected distances in addition to parallaxes. As in the case of VLM10, the present paper bases its bias-correction method on empirical models for the Galactic pulsar distribution and the luminosity function. These models do add an unquantified level of uncertainty to the analysis, but can easily be updated as our knowledge about the pulsar population grows through pulsar surveys. The evaluation of $\mathrm{H} \mathrm{I}$ distance limits is presented in Section 2, and the likelihood analysis to correct for the biases is derived in Section 3. Biascorrected parallaxes and distances are given in Tables 1 and 2 and a summarizing discussion is given in Section 4.

\section{H I KINEMATIC DISTANCES}

\subsection{Source Selection and H I Kinematic Distance Limit Determination}

FW90 established standard techniques for the extraction of reliable pulsar kinematic distance limits. Specifically, they 
Table 1

Pulsar H i Distance Limits and Lutz-Kelker-bias Corrected Distances and Parallaxes

\begin{tabular}{|c|c|c|c|c|c|c|c|c|c|}
\hline \multicolumn{2}{|c|}{ Pulsar Name } & \multirow[t]{2}{*}{ Association } & \multirow{2}{*}{$\begin{array}{l}\varpi_{\text {meas }} \\
\text { (mas) }\end{array}$} & \multirow{2}{*}{$\begin{array}{l}D_{\text {low }} \\
(\mathrm{kpc})\end{array}$} & \multirow{2}{*}{$\begin{array}{c}D_{\text {up }} \\
(\mathrm{kpc})\end{array}$} & \multirow{2}{*}{$\begin{array}{l}S_{1400} \\
(\mathrm{mJy})\end{array}$} & \multirow{2}{*}{$\begin{array}{l}\varpi_{\text {Corr }} \\
\text { (mas) }\end{array}$} & \multirow{2}{*}{$\begin{array}{l}D_{\text {Corr }} \\
(\mathrm{kpc})\end{array}$} & \multirow[t]{2}{*}{$\operatorname{Ref}^{\mathrm{a}}$} \\
\hline $\mathrm{J} 2000$ & B1950 & & & & & & & & \\
\hline J0141+6009 & B0138+59 & $\cdots$ & $\ldots$ & $2.6 \pm 0.7$ & $2.9 \pm 0.7$ & 4.5 & $0.30_{-0.05}^{+0.07}$ & $2.3 \pm 0.7$ & (1) \\
\hline J0332+5434 & В0329+54 & $\ldots$ & $0.94 \pm 0.11$ & $1.7 \pm 0.7$ & $2.0 \pm 0.8$ & 203 & $0.8 \pm 0.1$ & $1.0 \pm 0.1$ & $(1,21)$ \\
\hline J0358+5413 & B0355+54 & $\ldots$ & $0.91 \pm 0.16$ & $1.4 \pm 0.7$ & $2.2 \pm 0.9$ & 23 & $0.7 \pm 0.2$ & $1.0_{-0.1}^{+0.2}$ & $(1,22)$ \\
\hline J0738-4042 & B0736-40 & $\ldots$ & $\ldots$ & $2.1 \pm 0.6$ & $\ldots$ & 80 & $0.3 \pm 0.1$ & $1.6 \pm 0.8$ & (2) \\
\hline J0742-2822 & B0740-28 & $\ldots$ & $\ldots$ & $2.0 \pm 0.6$ & $6.9 \pm 0.8$ & 15 & $0.16_{-0.03}^{+0.07}$ & $2.0_{-0.8}^{+1.0}$ & (3) \\
\hline J0837-4135 & B0835-41 & $\ldots$ & $\ldots$ & $1.8 \pm 0.8$ & $6.0 \pm 0.7$ & 16 & $0.18_{-0.03}^{+0.06}$ & $1.5_{-0.9}^{+1.2}$ & (2) \\
\hline J0908-4913 & B0906-49 & $\ldots$ & $\ldots$ & $2.4 \pm 1.6$ & $6.7 \pm 0.7$ & 10.0 & $0.16_{-0.02}^{+0.05}$ & $1.0_{-0.7}^{+1.7}$ & (3) \\
\hline J0942-5552 & B0940-55 & $\ldots$ & $\ldots$ & $\ldots$ & $7.5 \pm 0.7$ & 10.0 & $0.16_{-0.03}^{+0.11}$ & $0.3_{-0.2}^{+0.8}$ & (2) \\
\hline J1001-5507 & B0959-54 & $\ldots$ & $\ldots$ & $\ldots$ & $6.9 \pm 0.7$ & 6.3 & $0.16_{-0.03}^{+0.08}$ & $0.3_{-0.3}^{+1.1}$ & (3) \\
\hline J1048-5832 & B $1046-58$ & $\ldots$ & $\ldots$ & $2.5 \pm 0.5$ & $5.6 \pm 0.8$ & 6.5 & $0.18_{-0.03}^{+0.05}$ & $2.9_{-0.7}^{+1.2}$ & (2) \\
\hline $\mathrm{J} 1056-6258$ & B $1054-62$ & $\ldots$ & $\ldots$ & $2.5 \pm 0.5$ & $2.9 \pm 0.5$ & 21 & $0.33_{-0.05}^{+0.06}$ & $2.4 \pm 0.5$ & (3) \\
\hline J1124-5916 & $\ldots$ & SNR G292.0+1.8 core & $\ldots$ & $3.2 \pm 2.0$ & $\ldots$ & 0.08 & $0.08_{-0.02}^{+0.04}$ & $5_{-2}^{+3}$ & $(4)^{b}$ \\
\hline J1141-6545 & $\ldots$ & $\ldots$ & $\ldots$ & $3.7 \pm 1.7$ & $\ldots$ & 3.3 & $0.12_{-0.04}^{+0.06}$ & $3 \pm 2$ & $(5)^{\mathrm{b}}$ \\
\hline$J 1157-6224$ & B $1154-62$ & $\ldots$ & $\ldots$ & $3.8 \pm 1.4$ & $9.0 \pm 0.6$ & 5.9 & $0.12_{-0.01}^{+0.03}$ & $4 \pm 2$ & (2) \\
\hline J1224-6407 & B1221-63 & $\ldots$ & $\ldots$ & $4.3 \pm 1.4$ & $11.4 \pm 0.7$ & 3.9 & $0.10_{-0.01}^{+0.03}$ & $4 \pm 2$ & (2) \\
\hline$J 1243-6423$ & B1240-64 & $\ldots$ & $\ldots$ & $4.5 \pm 1.9$ & $11.5 \pm 0.7$ & 13 & $0.10_{-0.01}^{+0.04}$ & $2 \pm 2$ & (1) \\
\hline J1326-5859 & B $1323-58$ & $\ldots$ & $\ldots$ & $3.0 \pm 1.0$ & $\ldots$ & 9.9 & $0.12_{-0.04}^{+0.07}$ & $3_{-1}^{+2}$ & (6) \\
\hline$J 1327-6222$ & B $1323-62$ & $\ldots$ & $\ldots$ & $5.1 \pm 1.7$ & $11.8 \pm 0.6$ & 16.0 & $0.093_{-0.009}^{+0.031}$ & $4 \pm 2$ & (1) \\
\hline J1359-6038 & B1356-60 & $\ldots$ & $\ldots$ & $5.6 \pm 1.7$ & $\ldots$ & 7.6 & $0.09_{-0.03}^{+0.04}$ & $5 \pm 2$ & (1) \\
\hline$J 1401-6357$ & B $1358-63$ & $\ldots$ & $\ldots$ & $1.6 \pm 0.5$ & $2.7 \pm 0.7$ & 6.2 & $0.31_{-0.06}^{+0.09}$ & $1.8_{-0.6}^{+0.7}$ & (2) \\
\hline$J 1453-6413$ & B1449-64 & $\ldots$ & $\ldots$ & $2.5 \pm 0.5$ & $\ldots$ & 14.0 & $0.13_{-0.04}^{+0.09}$ & $2.8_{-0.8}^{+1.3}$ & (3) \\
\hline J1513-5908 & B $1509-58$ & SNR G320.4-01.2 & $\ldots$ & $3.8 \pm 0.5$ & $6.6 \pm 1.4$ & 0.94 & $0.14_{-0.02}^{+0.04}$ & $4.4_{-0.8}^{+1.3}$ & (7) \\
\hline J1559-4438 & B1556-44 & $\ldots$ & $0.384 \pm 0.081$ & $2.0 \pm 0.5$ & $\ldots$ & 40 & $0.32_{-0.08}^{+0.07}$ & $2.3_{-0.3}^{+0.5}$ & $(3,23)$ \\
\hline$J 1600-5044$ & B $1557-50$ & $\ldots$ & $\ldots$ & $6.4 \pm 0.5$ & $18.2 \pm 1.2$ & 17.0 & $0.08_{-0.02}^{+0.03}$ & $6.9_{-0.9}^{+1.9}$ & (8) \\
\hline $\mathrm{J} 1602-5100$ & B $1558-50$ & $\ldots$ & $\ldots$ & $7.4 \pm 0.5$ & $9.4 \pm 0.4$ & 5.7 & $0.113_{-0.007}^{+0.013}$ & $8.0_{-0.7}^{+0.9}$ & (8) \\
\hline J1644-4559 & B1641-45 & $\ldots$ & $\ldots$ & $4.2 \pm 0.3$ & $5.0 \pm 0.3$ & 310 & $0.21 \pm 0.02$ & $4.5 \pm 0.4$ & (1) \\
\hline$J 1651-4246$ & B $1648-42$ & $\ldots$ & $\ldots$ & $4.8 \pm 0.3$ & $\ldots$ & 16.0 & $0.08_{-0.02}^{+0.04}$ & $5.2_{-0.6}^{+2.1}$ & (9) \\
\hline $\mathrm{J} 1707-4053$ & B $1703-40$ & $\ldots$ & $\ldots$ & $3.8 \pm 0.5$ & $\ldots$ & 7.2 & $0.08_{-0.02}^{+0.04}$ & $4_{-1}^{+2}$ & (9) \\
\hline J1709-4429 & B1706-44 & $\ldots$ & $\ldots$ & $2.4 \pm 0.6$ & $3.2 \pm 0.4$ & 7.3 & $0.31_{-0.04}^{+0.05}$ & $2.6_{-0.6}^{+0.5}$ & (3) \\
\hline $\mathrm{J} 1721-3532$ & B1718-35 & $\ldots$ & $\ldots$ & $4.4 \pm 0.5$ & $5.2 \pm 0.6$ & 11.0 & $0.19 \pm 0.02$ & $4.6 \pm 0.6$ & (9) \\
\hline J1740-3015 & B1737-30 & $\ldots$ & $\ldots$ & $\ldots$ & $5.5 \pm 0.6$ & 6.4 & $0.20_{-0.03}^{+0.07}$ & $0.4_{-0.3}^{+1.7}$ & (8) \\
\hline J1745-3040 & B1742-30 & $\ldots$ & $\ldots$ & $\ldots$ & $5.5 \pm 0.6$ & 13.0 & $0.20_{-0.03}^{+0.08}$ & $0.2_{-0.2}^{+1.1}$ & (8) \\
\hline J1752-2806 & B1749-28 & $\ldots$ & $\ldots$ & $0.125 \pm 0.025$ & $\ldots$ & 18.0 & $0.08 \pm 0.03^{\mathrm{c}}$ & $0.2_{-0.1}^{+1.1}$ & $(1,10)$ \\
\hline J1801-2304 & B1758-23 & $\ldots$ & $\ldots$ & $3.5 \pm 0.9$ & $6.9 \pm 0.1$ & 2.2 & $0.149_{-0.005}^{+0.033}$ & $4 \pm 1$ & (11) \\
\hline J1803-2137 & B1800-21 & SNR G8.7-0.1 & $\ldots$ & $4.0 \pm 0.6$ & $4.9 \pm 0.3$ & 7.6 & $0.21 \pm 0.02$ & $4.4_{-0.6}^{+0.5}$ & (1) \\
\hline J1807-0847 & B1804-08 & $\ldots$ & $\ldots$ & $1.5 \pm 0.7$ & $\ldots$ & 15.0 & $0.11_{-0.03}^{+0.10}$ & $1.5_{-0.9}^{+1.2}$ & (1) \\
\hline$\ldots$ & SGR $1806-20$ & radioflare 2005 & $\ldots$ & $6.2 \pm 0.1$ & $\ldots$ & $\ldots$ & $0.06_{-0.01}^{+0.02}$ & $13_{-3}^{+4}$ & (12) \\
\hline XTE J1810-197 & $\ldots$ & radioflare 2006 & $\ldots$ & $3.4 \pm 0.6$ & $3.4 \pm 0.6$ & $\ldots$ & $0.25_{-0.03}^{+0.04}$ & $3.6 \pm 0.5$ & $(13)^{d}$ \\
\hline J1820-0427 & B1818-04 & $\ldots$ & $\ldots$ & $\ldots$ & $1.6 \pm 0.5$ & 6.1 & $0.5_{-0.1}^{+0.2}$ & $0.3_{-0.2}^{+0.6}$ & (1) \\
\hline $\mathrm{J} 1823+0550$ & B1821+05 & $\ldots$ & $\ldots$ & $1.6 \pm 0.5$ & $\ldots$ & 1.7 & $0.13_{-0.04}^{+0.09}$ & $2.0_{-0.8}^{+1.3}$ & (1) \\
\hline J1824-1945 & B1821-19 & $\ldots$ & $\ldots$ & $3.2 \pm 0.5$ & $\ldots$ & 4.9 & $0.09_{-0.02}^{+0.07}$ & $3.7_{-0.9}^{+1.6}$ & (8) \\
\hline J1825-0935 & B1822-09 & $\ldots$ & $\ldots$ & $\ldots$ & $1.9 \pm 0.4$ & 12.0 & $0.5_{-0.1}^{+0.2}$ & $0.3_{-0.2}^{+0.7}$ & (8) \\
\hline J1832-0827 & B1829-08 & $\ldots$ & $\ldots$ & $4.7 \pm 0.3$ & $5.8 \pm 0.3$ & 2.1 & $0.18_{-0.01}^{+0.02}$ & $5.2_{-0.4}^{+0.5}$ & (1) \\
\hline J1833-0827 & B1830-08 & $\ldots$ & $\ldots$ & $4.0 \pm 0.4$ & $5.3 \pm 0.3$ & 3.6 & $0.20 \pm 0.02$ & $4.5 \pm 0.5$ & (9) \\
\hline J1833-1034 & $\ldots$ & SNR G21.5-0.9 & $\ldots$ & $4.0 \pm 0.3$ & $4.1 \pm 0.3$ & 0.071 & $0.24 \pm 0.02$ & $4.1 \pm 0.3$ & $(14)^{\mathrm{b}, \mathrm{e}}$ \\
\hline AXP 1E1841-045 & $\ldots$ & SNR Kes73 & $\ldots$ & $7.5 \pm 1.0$ & $10.2 \pm 0.3$ & $\ldots$ & $0.102_{-0.005}^{+0.012}$ & $9.6_{-1.4}^{+0.6}$ & $(15)^{\mathrm{d}, \mathrm{f}}$ \\
\hline $\mathrm{J} 1846-0258$ & $\ldots$ & SNR Kes75 & $\ldots$ & $5.5 \pm 0.4$ & $5.9 \pm 0.5$ & $\ldots$ & $0.17 \pm 0.01$ & $5.8_{-0.4}^{+0.5}$ & $(16)^{\mathrm{b}, \mathrm{e}}$ \\
\hline $\mathrm{J} 1848-0123$ & B1845-01 & $\ldots$ & $\ldots$ & $4.2 \pm 0.4$ & $4.8 \pm 0.4$ & 8.6 & $0.21 \pm 0.02$ & $4.4 \pm 0.4$ & (1) \\
\hline $\mathrm{J} 1852+0031$ & B1849+00 & $\ldots$ & $\ldots$ & $7.1 \pm 1.2$ & $16.6 \pm 0.9$ & 2.2 & $0.070_{-0.009}^{+0.025}$ & $8 \pm 2$ & (1) \\
\hline $\mathrm{J} 1857+0212$ & B1855+02 & $\ldots$ & $\ldots$ & $6.9 \pm 1.3$ & $\ldots$ & 1.6 & $0.08_{-0.02}^{+0.03}$ & $8 \pm 2$ & (1) \\
\hline $\mathrm{J} 1857+0943$ & B1855+09 & $\ldots$ & $1.1 \pm 0.2$ & $1.6 \pm 0.5$ & $2.0 \pm 0.4$ & 5 & $0.6_{-0.1}^{+0.2}$ & $0.9 \pm 0.2$ & $(1,24)$ \\
\hline $\mathrm{J} 1901+0331$ & B1859+03 & $\ldots$ & $\ldots$ & $6.8 \pm 1.4$ & $15.1 \pm 0.7$ & 4.2 & $0.075_{-0.008}^{+0.027}$ & $7 \pm 2$ & (1) \\
\hline $\mathrm{J} 1901+0716$ & B1859+07 & $\ldots$ & $\ldots$ & $2.8 \pm 0.5$ & $4.7 \pm 0.8$ & 0.9 & $0.20_{-0.03}^{+0.05}$ & $3.4_{-0.7}^{+0.9}$ & (1) \\
\hline $\mathrm{J} 1902+0556$ & B1900+05 & $\ldots$ & $\ldots$ & $3.1 \pm 0.4$ & $4.3 \pm 0.5$ & 1.2 & $0.23_{-0.03}^{+0.04}$ & $3.6_{-0.5}^{+0.6}$ & (1) \\
\hline
\end{tabular}


Table 1

(Continued)

\begin{tabular}{|c|c|c|c|c|c|c|c|c|c|}
\hline \multicolumn{2}{|c|}{ Pulsar Name } & \multirow[t]{2}{*}{ Association } & \multirow{2}{*}{$\begin{array}{l}\varpi_{\text {meas }} \\
\text { (mas) }\end{array}$} & \multirow{2}{*}{$\begin{array}{l}D_{\text {low }} \\
(\mathrm{kpc})\end{array}$} & \multirow{2}{*}{$\begin{array}{c}D_{\text {up }} \\
(\mathrm{kpc})\end{array}$} & \multirow{2}{*}{$\begin{array}{l}S_{1400} \\
(\mathrm{mJy})\end{array}$} & \multirow{2}{*}{$\begin{array}{l}\varpi_{\text {Corr }} \\
\text { (mas) }\end{array}$} & \multirow{2}{*}{$\begin{array}{l}D_{\text {Corr }} \\
(\mathrm{kpc})\end{array}$} & \multirow[t]{2}{*}{$\operatorname{Ref}^{\mathrm{a}}$} \\
\hline J2000 & B1950 & & & & & & & & \\
\hline $\mathrm{J} 1902+0615$ & B1900+06 & $\ldots$ & $\ldots$ & $6.5 \pm 1.4$ & $15.8 \pm 0.8$ & 1.1 & $0.071_{-0.007}^{+0.024}$ & $7_{-2}^{+3}$ & (1) \\
\hline $\mathrm{J} 1903+0135$ & B1900+01 & $\ldots$ & $\ldots$ & $2.8 \pm 0.4$ & $4.0 \pm 0.4$ & 1.1 & $0.26_{-0.03}^{+0.04}$ & $3.3_{-0.5}^{+0.6}$ & (1) \\
\hline $\mathrm{J} 1906+0641$ & B1904+06 & $\ldots$ & $\ldots$ & $6.5 \pm 1.5$ & $14.0 \pm 0.5$ & 1.7 & $0.077_{-0.006}^{+0.026}$ & $7 \pm 2$ & (1) \\
\hline $\mathrm{J} 1909+0254$ & B $1907+02$ & $\ldots$ & $\ldots$ & $3.8 \pm 0.5$ & $\ldots$ & 0.63 & $0.09_{-0.03}^{+0.05}$ & $4.5_{-0.9}^{+2.2}$ & (17) \\
\hline $\mathrm{J} 1909+1102$ & B1907+10 & $\ldots$ & $\ldots$ & $4.3 \pm 0.6$ & $6.0 \pm 1.6$ & 1.9 & $0.14_{-0.03}^{+0.04}$ & $4.8_{-0.8}^{+1.1}$ & (1) \\
\hline J1915+1009 & B1913+10 & $\ldots$ & $\ldots$ & $6.0 \pm 1.5$ & $14.5 \pm 0.8$ & 1.3 & $0.077_{-0.009}^{+0.027}$ & $7 \pm 2$ & (1) \\
\hline $\mathrm{J} 1916+1312$ & B1914+13 & $\ldots$ & $\ldots$ & $4.0 \pm 0.7$ & $5.7 \pm 1.7$ & 1.2 & $0.14_{-0.03}^{+0.04}$ & $4.5_{-0.9}^{+1.2}$ & (1) \\
\hline $\mathrm{J} 1917+1353$ & B1915+13 & $\ldots$ & $\ldots$ & $4.8 \pm 1.0$ & $5.7 \pm 1.7$ & 1.9 & $0.14 \pm 0.03$ & $5 \pm 1$ & (1) \\
\hline $\mathrm{J} 1921+2153$ & B1919+21 & $\ldots$ & $\ldots$ & $\ldots$ & $2.8 \pm 1.2$ & 6 & $0.29_{-0.08}^{+0.15}$ & $0.3_{-0.2}^{+0.8}$ & (1) \\
\hline $\mathrm{J} 1922+2110$ & B1920+21 & $\ldots$ & $\ldots$ & $4.8 \pm 1.8$ & $16.2 \pm 1.0$ & 1.4 & $0.08_{-0.02}^{+0.03}$ & $4 \pm 2$ & (17) \\
\hline$J 1926+1648$ & B1924+16 & $\ldots$ & $\ldots$ & $5.2 \pm 1.8$ & $14.9 \pm 0.8$ & 1.3 & $0.075_{-0.008}^{+0.028}$ & $6_{-2}^{+3}$ & (17) \\
\hline J1932+1059 & B1929+10 & $\ldots$ & $2.77 \pm 0.07$ & $\ldots$ & $1.6 \pm 0.5$ & 36 & $0.9_{-0.3}^{+1.0}$ & $0.31_{-0.06}^{+0.09}$ & $(1,22)$ \\
\hline $\mathrm{J} 1932+2020$ & B $1929+20$ & $\ldots$ & $\ldots$ & $4.8 \pm 1.8$ & $14.9 \pm 0.9$ & 1.2 & $0.076_{-0.009}^{+0.029}$ & $5_{-2}^{+3}$ & (1) \\
\hline J1930+1852 & $\ldots$ & PWN G54.1+0.3 & $\ldots$ & $5.0 \pm 1.8$ & $12.6 \pm 0.6$ & 0.06 & $0.085_{-0.007}^{+0.020}$ & $7_{-2}^{+3}$ & $(18)^{e, f}$ \\
\hline $\mathrm{J} 1932+2220$ & B $1930+22$ & $\ldots$ & $\ldots$ & $10.4 \pm 0.6$ & $13.7 \pm 0.7$ & 1.2 & $0.081_{-0.007}^{+0.010}$ & $10.9_{-0.8}^{+1.3}$ & (1) \\
\hline J1935+1616 & B1933+16 & $\ldots$ & $0.22_{-0.12}^{+0.08}$ & $5.2 \pm 1.7$ & $\ldots$ & 42 & $0.13_{-0.04}^{+0.05}$ & $3.7_{-0.8}^{+1.3}$ & $(1,25)$ \\
\hline J1939+2134 & B1937+21 & $\ldots$ & $0.13 \pm 0.07$ & $4.6 \pm 1.9$ & $14.8 \pm 0.9$ & 10 & $0.08_{-0.01}^{+0.03}$ & $5_{-1}^{+2}$ & $(1,24)$ \\
\hline J1946+1805 & B1944+17 & $\ldots$ & $\ldots$ & $\ldots$ & $1.9 \pm 0.7$ & 10 & $0.4_{-0.1}^{+0.2}$ & $0.3_{-0.2}^{+0.6}$ & (1) \\
\hline $\mathrm{J} 1952+3252$ & B1951+32 & SNR CTB80 & $\ldots$ & $3.1 \pm 2.0$ & $\ldots$ & 1.0 & $0.11_{-0.03}^{+0.06}$ & $3 \pm 2$ & $(19)^{f, g}$ \\
\hline J2004+3137 & B2002+31 & $\ldots$ & $\ldots$ & $7.0 \pm 0.7$ & $12.0 \pm 0.7$ & 1.8 & $0.092_{-0.009}^{+0.023}$ & $8_{-1}^{+2}$ & (1) \\
\hline J2018+2839 & B2016+28 & $\ldots$ & $1.03 \pm 0.10$ & $3.2 \pm 2.1$ & $\ldots$ & 30 & $0.9 \pm 0.1$ & $0.98_{-0.09}^{+0.11}$ & $(1,21)$ \\
\hline $\mathrm{J} 2022+2854$ & B2020+28 & $\ldots$ & $0.37 \pm 0.12$ & $3.1 \pm 2.1$ & $\ldots$ & 38 & $0.22_{-0.07}^{+0.10}$ & $2.1_{-0.4}^{+0.6}$ & $(1,21)$ \\
\hline$J 2113+4644$ & B2111+46 & $\ldots$ & $\ldots$ & $4.3 \pm 0.8$ & $6.5 \pm 0.7$ & 19 & $0.17_{-0.02}^{+0.03}$ & $4 \pm 1$ & (1) \\
\hline J2257+5909 & B $2255+58$ & $\ldots$ & $\ldots$ & $3.3 \pm 0.7$ & $\ldots$ & 9.2 & $0.13_{-0.04}^{+0.08}$ & $3 \pm 1$ & (1) \\
\hline$\ldots$ & AXP 1E2259+586 & SNR CTB109 & $\ldots$ & $4.0 \pm 0.8$ & $4.0 \pm 0.8^{\mathrm{h}}$ & $\ldots$ & $0.21_{-0.03}^{+0.04}$ & $4.1 \pm 0.7$ & (20) \\
\hline $\mathrm{J} 2321+6024$ & B2319+60 & $\ldots$ & $\ldots$ & $2.6 \pm 0.6$ & $\ldots$ & 12 & $0.13_{-0.05}^{+0.10}$ & $2.7_{-0.9}^{+1.2}$ & (1) \\
\hline
\end{tabular}

Notes.

a References: (1) Frail \& Weisberg 1990; (2) Johnston et al. 1996; (3) Koribalski et al. 1995; (4) Gaensler \& Wallace 2003; (5) Ord et al. 2002; (6) Saravanan et al. 1996; (7) Gaensler et al. 1999; (8) Johnston et al. 2001; (9) Weisberg et al. 1995; (10) Johnston et al. 2001; (11) Frail et al. 1993 and Frail 1993, private communication; (12) McClure-Griffiths \& Gaensler 2005; (13) Minter et al. 2008; (14) Tian \& Leahy 2008b; (15) Tian \& Leahy 2008a; (16) Leahy \& Tian 2008; (17) Weisberg et al. 2008; (18) Leahy et al. 2008; (19) Strom \& Stappers 2000; (20) Tian et al. 2010; (21) Brisken et al. 2002; (22) Chatterjee et al. 2004; (23) Deller et al. 2009c; (24) Verbiest et al. 2009; (25) Chatterjee et al. 2009.

${ }^{\mathrm{b}}$ Distance limit uncertainty is derived by the current authors.

${ }^{c}$ PSR J1752-2806 (B1749-28) has a secondary optimum in its parallax, at $0.15_{-0.02}^{+0.03}$ mas.

d Original authors cited multiple distances derived from multiple rotation curves; we choose the standard flat model.

${ }^{\mathrm{e}}$ Reverted flat rotation model to old Galactic constants $\left(R_{0}, \Theta_{0}\right)=\left(8.5 \mathrm{kpc}, 220 \mathrm{~km} \mathrm{~s}^{-1}\right)$.

${ }^{\mathrm{f}}$ Distance limit and uncertainty are derived by the current authors. See notes in body of paper.

g The original authors did not publish the absorption spectrum at velocities enabling a $D_{\text {up }}$ measurement.

${ }^{\text {h }}$ Upper limit based on CO emission from a molecular cloud associated with SNR CTB109.

defined the bound of $T_{\mathrm{b}} \geqslant 35 \mathrm{~K}$ on the brightness temperature of $\mathrm{H}$ I emission used for deriving upper distance limits, ${ }^{4}$ pointing out that weaker emission would not be expected to result in significant absorption. Second, they re-evaluated distance limits based on old Galactic models and rotation curves, defaulting to the IAU values for the distance of the solar system to the center of the Galaxy $\left[R_{0}=8.5 \mathrm{kpc}\right]$ and the Galactic rotation velocity in the solar system neighborhood $\left[\Theta_{0}=220 \mathrm{~km} \mathrm{~s}^{-1}\right]$ (Kerr \& Lynden-Bell 1986), and using the flat rotation curve of Fich et al. (1989). In converting velocities to distances, furthermore, they assumed an uncertainty of $7 \mathrm{~km} \mathrm{~s}^{-1}$ because of known random motions of that order (Dickey \& Lockman 1990). Finally, in the Perseus arm, with its well-known spiral

\footnotetext{
4 They also note this bound can be relaxed depending on the sensitivity of the
} observation, provided the optical depth for the emission is 0.3 or higher. shock, they either used independent distance tracers or applied the approximation proposed by Joncas et al. (1989), which states the global rotation curve can be applied (near $G_{1}=130^{\circ}$ ) provided the measured $\mathrm{H}$ I velocities are decreased by a factor of 1.6. Most investigations since then have used these same criteria and so does the present paper, with a few exceptions as listed below.

In the current work, we present a uniformly determined sample of neutron star H I kinematic upper and lower distance estimates by finding all such efforts in the literature, and then applying the FW90 criteria to any published data that have not previously been analyzed with that procedure. If the cited authors made a good case for a non-flat rotation curve (e.g, in the direction of the Galactic bar, the $3 \mathrm{kpc}$ arm, or the Perseus arm shock), we maintain their curve in our analysis. If, however, the original authors used a flat rotation curve but non-IAU Galactic 
Table 2

Pulsar Parallax Measurements and Lutz-Kelker-bias-corrected Distances and Parallaxes for Pulsars without H I Limits But with Parallax Measurements Only

\begin{tabular}{|c|c|c|c|c|c|c|}
\hline \multicolumn{2}{|c|}{ Pulsar Nsame } & \multirow{2}{*}{$\begin{array}{c}\varpi_{\text {meas }} \\
\text { (mas) }\end{array}$} & \multirow{2}{*}{$\begin{array}{l}S_{1400} \\
(\mathrm{mJy})\end{array}$} & \multirow{2}{*}{$\begin{array}{l}\varpi_{\text {Corr }} \\
\text { (mas) }\end{array}$} & \multirow{2}{*}{$\begin{array}{l}D_{\text {Corr }} \\
(\mathrm{kpc})\end{array}$} & \multirow[t]{2}{*}{ Ref. } \\
\hline $\mathrm{J} 2000$ & B1950 & & & & & \\
\hline $\mathrm{J} 0030+0451$ & $\ldots$ & $3.3 \pm 0.9$ & 0.6 & $1.6_{-0.8}^{+1.0}$ & $0.28_{-0.06}^{+0.10}$ & Lommen et al. $(2006,2000)$ \\
\hline J0034-0721 & B0031-07 & $0.93_{-0.07}^{+0.08}$ & 11 & $0.93_{-0.07}^{+0.08}$ & $1.03 \pm 0.08$ & Chatterjee et al. (2009) \\
\hline J0108-1431 & $\ldots$ & $4.2 \pm 1.4$ & 1.0 & $1.4_{-0.7}^{+1.4}$ & $0.21_{-0.05}^{+0.09}$ & Deller et al. (2009b) \\
\hline $\mathrm{J} 0139+5814$ & B0136+57 & $0.37 \pm 0.04$ & 4.6 & $0.37 \pm 0.04$ & $2.6_{-0.2}^{+0.3}$ & Chatterjee et al. (2009) \\
\hline J0437-4715 & $\ldots$ & $6.396 \pm 0.054$ & 142 & $6.39 \pm 0.05$ & $0.156 \pm 0.001$ & Deller et al. (2008) \\
\hline J0452-1759 & B0450-18 & $0.64_{-0.6}^{+1.4}$ & 5.3 & $0.7_{-0.3}^{+0.6}$ & $0.4_{-0.1}^{+0.2}$ & Chatterjee et al. (2009) \\
\hline J0454+5543 & B $0450+55$ & $0.84_{-0.05}^{+0.04}$ & 13 & $0.84_{-0.05}^{+0.04}$ & $1.18_{-0.05}^{+0.07}$ & Chatterjee et al. (2009) \\
\hline J0538+2817 & $\ldots$ & $0.72_{-0.09}^{+0.12}$ & 1.9 & $0.69_{-0.09}^{+0.11}$ & $1.3 \pm 0.2$ & Chatterjee et al. (2009); Lewandowski et al. (2004) \\
\hline J0613-0200 & $\ldots$ & $0.80 \pm 0.35$ & 1.4 & $0.4_{-0.2}^{+0.3}$ & $0.9_{-0.2}^{+0.4}$ & Verbiest et al. (2009) \\
\hline J0630-2834 & B0628-28 & $3.0 \pm 0.4$ & 23 & $2.8 \pm 0.4$ & $0.32_{-0.04}^{+0.05}$ & Deller et al. (2009b) \\
\hline J0633+1746 & $\ldots$ & $4.0 \pm 1.3$ & $\ldots$ & $0.2_{-0.1}^{+0.5}$ & $0.25_{-0.08}^{+0.23}$ & Faherty et al. (2007) \\
\hline J0659+1414 & B0656+14 & $3.47 \pm 0.36$ & 3.7 & $3.3 \pm 0.4$ & $0.28 \pm 0.03$ & Brisken et al. (2003) \\
\hline J0720-3125 & $\ldots$ & $2.77 \pm 0.89$ & $\ldots$ & $0.2_{-0.1}^{+0.8}$ & $0.4_{-0.1}^{+0.3}$ & Kaplan et al. (2007) \\
\hline J0737-3039A & $\ldots$ & $0.87 \pm 0.14$ & 1.6 & $0.80 \pm 0.14$ & $1.1_{-0.1}^{+0.2}$ & Deller et al. (2009a); Burgay et al. (2006) \\
\hline $\mathrm{J} 0751+1807$ & $\ldots$ & $1.6 \pm 0.8$ & 3.23 & $0.6_{-0.3}^{+0.6}$ & $0.4_{-0.1}^{+0.2}$ & Nice et al. (2005) \\
\hline J0814+7429 & B0809+74 & $2.31 \pm 0.04$ & 10 & $2.31 \pm 0.04$ & $0.432_{-0.007}^{+0.008}$ & Brisken et al. (2002) \\
\hline J0820-1350 & B0818-13 & $0.51_{-0.04}^{+0.03}$ & 7 & $0.51_{-0.04}^{+0.03}$ & $1.9 \pm 0.1$ & Chatterjee et al. (2009) \\
\hline $\mathrm{J} 0826+2637$ & B0823+26 & $2.8 \pm 0.6$ & 10 & $2.4 \pm 0.6$ & $0.32_{-0.05}^{+0.08}$ & Gwinn et al. (1986) \\
\hline J0835-4510 & B $0833-45$ & $3.5 \pm 0.2$ & 1100 & $3.5 \pm 0.2$ & $0.28 \pm 0.02$ & Dodson et al. (2003); Backer \& Fisher (1974) \\
\hline J0922+0638 & B0919+06 & $0.82 \pm 0.13$ & 4.2 & $0.82_{-0.12}^{+0.13}$ & $1.1_{-0.1}^{+0.2}$ & Chatterjee et al. (2001) \\
\hline J0953+0755 & B0950+08 & $3.82 \pm 0.07$ & 84 & $3.82 \pm 0.07$ & $0.261 \pm 0.005$ & Brisken et al. (2002) \\
\hline $\mathrm{J} 1012+5307$ & $\ldots$ & $1.22 \pm 0.26$ & 3 & $1.11 \pm 0.25$ & $0.7_{-0.1}^{+0.2}$ & Lazaridis et al. (2009) \\
\hline $\mathrm{J} 1022+1001$ & $\ldots$ & $1.8 \pm 0.3$ & 3 & $1.7 \pm 0.3$ & $0.52_{-0.07}^{+0.09}$ & Verbiest et al. (2009) \\
\hline J1024-0719 & $\ldots$ & $1.9 \pm 0.4$ & 0.66 & $1.5 \pm 0.4$ & $0.49_{-0.08}^{+0.12}$ & Hotan et al. (2006) \\
\hline J1045-4509 & $\ldots$ & $3.3 \pm 1.9$ & 3 & $0.3_{-0.1}^{+0.4}$ & $0.23_{-0.07}^{+0.17}$ & Verbiest et al. (2009) \\
\hline J1136+1551 & B1133+16 & $2.80 \pm 0.16$ & 32 & $2.80 \pm 0.16$ & $0.35 \pm 0.02$ & Brisken et al. (2002) \\
\hline $\mathrm{J} 1239+2453$ & B1237+25 & $1.16 \pm 0.08$ & 10 & $1.16 \pm 0.08$ & $0.84+0.06-0.05$ & Brisken et al. (2002) \\
\hline $\mathrm{J} 1300+1240$ & B1257+12 & $1.3 \pm 0.4$ & 2 & $1.0_{-0.3}^{+0.4}$ & $0.6_{-0.1}^{+0.2}$ & Wolszczan et al. (2000) \\
\hline$J 1456-6843$ & B1451-68 & $2.2 \pm 0.3$ & 80 & $2.1 \pm 0.3$ & $0.43_{-0.05}^{+0.06}$ & Bailes et al. (1990); Manchester et al. (1980) \\
\hline $\mathrm{J} 1509+5531$ & B $1508+55$ & $0.47 \pm 0.03$ & 8 & $0.47 \pm 0.03$ & $2.1 \pm 0.1$ & Chatterjee et al. (2009) \\
\hline $\mathrm{J} 1537+1155$ & B1534+12 & $0.98 \pm 0.05$ & 0.6 & $0.97 \pm 0.05$ & $1.01 \pm 0.05$ & Stairs et al. (2002) \\
\hline J1543+0929 & B1541+09 & $0.13 \pm 0.02$ & 5.9 & $0.16 \pm 0.02$ & $5.9_{-0.5}^{+0.6}$ & Chatterjee et al. (2009) \\
\hline $\mathrm{J} 1600-3053$ & $\ldots$ & $0.20 \pm 0.15$ & 3.2 & $0.21_{-0.07}^{+0.10}$ & $2.4_{-0.6}^{+0.9}$ & Verbiest et al. (2009); Jacoby et al. (2007) \\
\hline J1643-1224 & $\cdots$ & $2.2 \pm 0.4$ & 4.8 & $1.9 \pm 0.4$ & $0.42_{-0.06}^{+0.09}$ & Verbiest et al. (2009) \\
\hline $\mathrm{J} 1713+0747$ & $\ldots$ & $0.94 \pm 0.05$ & 8 & $0.93 \pm 0.05$ & $1.05_{-0.05}^{+0.06}$ & Verbiest et al. (2009) \\
\hline J1744-1134 & $\ldots$ & $2.4 \pm 0.1$ & 3 & $2.4 \pm 0.1$ & $0.42 \pm 0.02$ & Verbiest et al. (2009) \\
\hline J1856-3754 & $\ldots$ & $6.2 \pm 0.6$ & $\ldots$ & $6.0 \pm 0.6$ & $0.16_{-0.01}^{+0.02}$ & van Kerkwijk \& Kaplan (2007) \\
\hline J1900-2600 & B1857-26 & $0.5 \pm 0.6$ & 13 & $0.3_{-0.1}^{+0.3}$ & $0.7_{-0.2}^{+0.4}$ & Fomalont et al. (1999) \\
\hline J1909-3744 & $\ldots$ & $0.79 \pm 0.02$ & 3 & $0.79 \pm 0.02$ & $1.26 \pm 0.03$ & Verbiest et al. (2009); Jacoby et al. (2003) \\
\hline $\mathrm{J} 2022+5154$ & B2021+51 & $0.50 \pm 0.07$ & 27 & $0.49 \pm 0.07$ & $1.8_{-0.2}^{+0.3}$ & Brisken et al. (2002) \\
\hline J2048-1616 & B2045-16 & $1.05_{-0.02}^{+0.03}$ & 13 & $1.05_{-0.02}^{+0.03}$ & $0.95_{-0.03}^{+0.02}$ & Chatterjee et al. (2009) \\
\hline $\mathrm{J} 2055+3630$ & B2053+36 & $0.17 \pm 0.03$ & 2.6 & $0.17 \pm 0.03$ & $5.0_{-0.6}^{+0.8}$ & Chatterjee et al. (2009) \\
\hline J2124-3358 & $\ldots$ & $3.1 \pm 0.6$ & 1.6 & $2.5_{-0.7}^{+0.6}$ & $0.30_{-0.05}^{+0.07}$ & Verbiest et al. (2009) \\
\hline J2129-5721 & $\ldots$ & $1.9 \pm 0.9$ & 1.4 & $0.5_{-0.3}^{+0.6}$ & $0.4_{-0.1}^{+0.2}$ & Verbiest et al. (2009) \\
\hline J2144-3933 & $\ldots$ & $6.05 \pm 0.56$ & 0.8 & $5.77 \pm 0.57$ & $0.16_{-0.01}^{+0.02}$ & Deller et al. (2009b) \\
\hline $\mathrm{J} 2145-0750$ & $\ldots$ & $1.6 \pm 0.3$ & 8 & $1.5 \pm 0.3$ & $0.57_{-0.08}^{+0.11}$ & Verbiest et al. (2009) \\
\hline $\mathrm{J} 2157+4017$ & B2154+40 & $0.28 \pm 0.06$ & 17 & $0.29 \pm 0.05$ & $2.9_{-0.4}^{+0.5}$ & Chatterjee et al. (2009) \\
\hline $\mathrm{J} 2313+4253$ & $\mathrm{~B} 2310+42$ & $0.93_{-0.07}^{+0.06}$ & 15 & $0.92_{-0.07}^{+0.06}$ & $1.06_{-0.06}^{+0.08}$ & Chatterjee et al. (2009) \\
\hline
\end{tabular}

constants, we reanalyze the kinematic distance limits, using the flat rotation curve and IAU constants. We note that Reid et al. (2009) obtain a Galactic rotation velocity that is larger than the IAU value at a significance of $95 \%$. More recent measurements by the same authors have increased the significance of this offset to close to $99 \%$ (M. J. Reid 2012, private communication). Based on Equation (2.21) of Damour \& Taylor (1991), we find that this could imply an overestimate of our $\mathrm{H}$ I distance limits by up to 
$\sim 20 \%$ (though generally much less), depending on Galactic longitude and the measured $\mathrm{H}$ I velocity.

In Table 1, we list the $\mathrm{H}$ i kinematic lower and upper distance limits, $D_{\text {low }}$ and $D_{\text {up }}$, respectively, and their uncertainties, $\sigma_{\text {low }}$ and $\sigma_{\text {up }}$, to conventional radio pulsars as well as to otherwise radio-quiet neutron stars with radio bursts, and to supernova remnants (SNRs) securely associated with various kinds of neutron stars. The values shown are from the stated authors' work, unless otherwise indicated in the table. If the original authors gave distance limits meeting our criteria, but neglected to derive uncertainties on these limits, then we do so ourselves according to the procedure laid out in FW90. In such cases, the table entry's reference shows a superscript $b$. If we judge that the listed authors' distance limits themselves need adjustment, then we do so and mark the entry's reference with a superscript $f$ and describe details of any such changes in Section 2.2. Two sources, marked with the superscript $d$, had two or more original sets of distance limits because the cited authors evaluated multiple Galactic rotation models without expressing a clear preference for one; in those cases, we select the one using the standard flat rotation curve, for overall consistency. (Note that in none of these cases the various rotation models provided significantly different results.)

\subsection{Notes on Individual Sources}

In the subsections below, we explain any adjustments to criteria that led to the originally published upper and/or lower distance limits. The values themselves are summarized in Table 1.

\subsubsection{SNR Kesteven 73 and AXP 1E1841-045}

Tian \& Leahy (2008a) performed an H I kinematic distance study of the SNR Kes 73, which is associated with AXP 1E1841-045. The authors showed that the SNR absorption extends to the tangent point, $7.5 \mathrm{kpc}$ distant, which marks the lower distance limit. They also made the case that the lack of absorption at $v=84 \mathrm{~km} \mathrm{~s}^{-1}$ sets an upper distance limit on the far side of the tangent point. We find these arguments compelling. However, we find that the flat rotation curve then indicates that $D_{\text {up }}=10.2 \mathrm{kpc}$, whereas Tian \& Leahy (2008a) quoted $D_{\text {up }}=9.8 \mathrm{kpc}$ for a flat rotation curve.

\subsubsection{PWN G54.1+0.3 and PSR J1930+1852}

Leahy et al. (2008) analyzed H i spectra of PWN G54.1+0.3, which is associated with PSR J1930+1852. We confirm that the lower distance limit is at the tangent point. While they place the upper distance limit at the solar circle on the far side of the Galaxy due to a lack of any negative velocity absorption, we instead adhere to the procedure of FW90, relaxing the limit to the distance corresponding to the first strong emission at negative velocities not showing absorption, i.e., at $v=-30 \mathrm{~km} \mathrm{~s}^{-1}$. After resetting the rotation curve to the flat model with IAU galactic constants, we then find that $\left(D_{\text {low }}, D_{\text {up }}\right)=(5.0,12.6) \mathrm{kpc}$.

\subsubsection{SNR CTB 80 and PSR B1951+32}

Strom \& Stappers (2000) measured the H I absorption spectrum of SNR CTB 80, which is associated with PSR B1951+32. There is significant absorption out to the tangent point, yielding $D_{\text {low }}=3.1 \mathrm{kpc}$. Unfortunately, the published absorption spectrum does not extend below $v=-15 \mathrm{~km} \mathrm{~s}^{-1}$, which is insufficient to establish a $D_{\text {up }}$ measurement.

\section{LUTZ-KELKER BIAS AND CORRECTIONS}

Lutz \& Kelker (1973) first presented the argument that because of the nonlinearity of sample volume with distance, objects are statistically more likely to be further away rather than closer by. Correction for this bias (which is related to the Malmquist bias but is more correctly named Lutz-Kelker bias, as discussed by VLM10) is relatively straightforward through a likelihood analysis that incorporates probabilities derived from a variety of possible measurements. Our derivation is similar to that of VLM10 but differs in a few fundamental areas. First, the primary focus of VLM10 was biases in parallax measurements, while our analysis considers both parallax and distance, which is a more natural quantity when dealing with $\mathrm{H}$ I distance limits. (Note that the conversion between parallax and distance is not a simple inversion in the case of finite uncertainties, as the transformation between these two quantities is nonlinear.) Second, where VLM10 applied a Bayesian analysis with prior information based on the pulsar luminosity and position in the Galaxy, we consider these quantities as measurements and hence have no need for prior information at all, which removes the Bayesian character of this analysis and leaves a straightforward likelihood analysis. Effectively, this is no more than an esthetic difference, however, which does not affect the results. Indeed, our approach could be considered Bayesian with a uniform prior. In particular, our analysis considers the following possible measurements:

1. a parallax measurement, $\varpi_{\text {meas }}$;

2. a lower H I distance limit, $D_{\text {low }}$;

3. an upper $\mathrm{H}$ I distance limit, $D_{\mathrm{up}}$;

4. the pulsar radio flux, $S$ (measured at or near an observing frequency of $1.4 \mathrm{GHz}$ ); and

5. the pulsar's Galactic position, $G_{1}, G_{\mathrm{b}}$.

Given a subset or all of these measurements and assuming no correlations between these values, we can determine the probability density function of the pulsar distance, $D$, through

$$
\begin{aligned}
& p\left(D \mid \varpi_{\text {meas }}, D_{\text {low }}, D_{\text {up }}, S, G_{1}, G_{\mathrm{b}}\right) \\
& =p\left(D \mid \varpi_{\text {meas }}\right) p\left(D \mid D_{\text {low }}\right) p\left(D \mid D_{\text {up }}\right) p(D \mid S) p\left(D \mid G_{1}, G_{\mathrm{b}}\right) .
\end{aligned}
$$

In the above equation (as in all equations throughout this paper), we only explicitly state dependence on parameters, while dependence on the uncertainties of said parameters is implied. In other words, where we write $p\left(D \mid \varpi_{\text {meas }}\right)$, we really mean $p\left(D \mid \varpi_{\text {meas }}, \sigma_{\varpi_{\text {meas }}}\right)$. In the following, these five terms will be derived; they will, respectively, be referred to as the parallax term, the lower $\mathrm{H}_{\mathrm{I}}$ limit term, the upper $\mathrm{H}_{\mathrm{I}}$ limit term, the luminosity term, and the volumetric or Galactic term.

\subsection{The Parallax Term, $p\left(D \mid \varpi_{\text {meas }}\right)$}

Given a measurement $\varpi_{\text {meas }}$ with uncertainty $\sigma_{\varpi}$ and assuming a Gaussian uncertainty distribution, the probability of the true parallax given the data is

$$
p\left(\varpi \mid \varpi_{\text {meas }}\right) \propto \frac{1}{\sqrt{2 \pi} \sigma_{\varpi}} \exp \left[-\frac{1}{2}\left(\frac{\varpi_{\text {meas }}-\varpi}{\sigma_{\varpi}}\right)^{2}\right] .
$$

Since $p(D)=|\partial \varpi / \partial D| p(\varpi) \propto p(\varpi) / D^{2}$, this means

$$
p\left(D \mid \varpi_{\text {meas }}\right) \propto \frac{1}{D^{2}} \exp \left[-\frac{1}{2}\left(\frac{\varpi_{\text {meas }}-1 / D}{\sigma_{\varpi}}\right)^{2}\right] .
$$


In the case of asymmetric uncertainties on parallax measurements (as given, e.g., by Chatterjee et al. 2009), we assume

$$
\begin{aligned}
& p\left(\varpi \mid \varpi_{\text {meas }}\right) \propto H\left(\varpi-\varpi_{\text {meas }}\right) \exp \left[-\frac{1}{2}\left(\frac{\varpi_{\text {meas }}-\varpi}{\sigma_{\varpi \text { up }}}\right)^{2}\right] \\
& \quad+H\left(\varpi_{\text {meas }}-\varpi\right) \exp \left[-\frac{1}{2}\left(\frac{\varpi_{\text {meas }}-\varpi}{\sigma_{\varpi \text { low }}}\right)^{2}\right]
\end{aligned}
$$

with $\varpi+\sigma_{\varpi \text { up }}$ and $\varpi-\sigma_{\varpi \text { low }}$, respectively, the upper and lower limits of the $1 \sigma$ interval of the measurement's probability density function, and with $H(x)$ the Heaviside step function, for which

$$
H(x)= \begin{cases}0 & \text { if } x<0 \\ 0.5 & \text { if } x=0 \\ 1 & \text { if } x>0\end{cases}
$$

For distance, as in the symmetric case, the extra factor of $D^{-2}$ is added, resulting in

$$
\begin{aligned}
& p\left(D \mid \varpi_{\text {meas }}\right) \\
& \propto \frac{1}{D^{2}} H\left(1 / D-\varpi_{\text {meas }}\right) \exp \left[-\frac{1}{2}\left(\frac{\varpi_{\text {meas }}-1 / D}{\sigma_{\varpi \text { up }}}\right)^{2}\right] \\
& +\frac{1}{D^{2}} H\left(\varpi_{\text {meas }}-1 / D\right) \exp \left[-\frac{1}{2}\left(\frac{\varpi_{\text {meas }}-1 / D}{\sigma_{\varpi \text { low }}}\right)^{2}\right] .
\end{aligned}
$$

\subsection{The H I Distance Limit Terms, $p\left(D \mid D_{\text {up }}, D_{\text {low }}\right)$}

Assuming the distance of the furthest absorbing $\mathrm{HI}_{\mathrm{I}}$ gas is determined to be $D_{\text {low }}$ with measurement uncertainty $\sigma_{\text {low }}$, then the probability distribution of the actual distance of the limiting gas is given (assuming Gaussian uncertainties) by

$$
p\left(d \mid D_{\text {low }}\right) \propto \frac{1}{\sqrt{2 \pi} \sigma_{\text {low }}} \exp \left[-\frac{1}{2}\left(\frac{D_{\text {low }}-d}{\sigma_{\text {low }}}\right)^{2}\right],
$$

where $d$ is the actual distance of the gas and hence the actual lower limit on the pulsar distance. This implies that for any pulsar distance $D$, we must have $D \geqslant d$. Hence, we derive the probability distribution for the pulsar's distance as

$$
p\left(D \mid D_{\text {low }}\right)=\int_{0}^{\infty} p(D \mid d) p\left(d \mid D_{\text {low }}\right) \mathrm{d} d,
$$

in which

$$
p(D \mid d) \propto H(D-d)
$$

with $H(x)$ being the Heaviside function, as defined above. We therefore have

$$
\begin{aligned}
p\left(D \mid D_{\text {low }}\right) & \propto \int_{0}^{\infty} H(D-d) p\left(d \mid D_{\text {low }}\right) \mathrm{d} d \\
& =\int_{0}^{D} p\left(d \mid D_{\text {low }}\right) \mathrm{d} d
\end{aligned}
$$

which results in

$$
p\left(D \mid D_{\text {low }}\right) \propto \frac{1}{2}\left[\operatorname{erf}\left(\frac{D_{\text {low }}}{\sqrt{2} \sigma_{\text {low }}}\right)-\operatorname{erf}\left(\frac{D_{\text {low }}-D}{\sqrt{2} \sigma_{\text {low }}}\right)\right],
$$

with $\operatorname{erf}(x)=(2 / \sqrt{\pi}) \int_{0}^{x} e^{-t^{2}} \mathrm{~d} t$ the error function.
Analogous to the above derivation, we have the probability distribution for the distance of the nearest gas not seen in absorption

$$
p\left(d \mid D_{\text {up }}\right) \propto \frac{1}{\sqrt{2 \pi} \sigma_{\text {up }}} \exp \left[-\frac{1}{2}\left(\frac{D_{\text {up }}-d}{\sigma_{\text {up }}}\right)^{2}\right] ;
$$

which is used in the probability distribution for the pulsar's distance as

$$
p\left(D \mid D_{\text {up }}\right)=\int_{0}^{\infty} p(D \mid d) p\left(d \mid D_{\text {up }}\right) \mathrm{d} d
$$

with

$$
p(D \mid d) \propto H(d-D)
$$

hence,

$$
\begin{aligned}
p\left(D \mid D_{\text {up }}\right) & \propto \int_{0}^{\infty} H(d-D) p\left(d \mid D_{\text {up }}\right) \mathrm{d} d \\
& =\int_{D}^{\infty} p\left(d \mid D_{\text {up }}\right) \mathrm{d} d
\end{aligned}
$$

which results in

$$
p\left(D \mid D_{\text {up }}\right) \propto \frac{1}{2}\left[\operatorname{erf}\left(\frac{D_{\text {up }}-D}{\sqrt{2} \sigma_{\text {up }}}\right)+1\right] .
$$

\subsection{The Galactic ("Volumetric”) Term, $p\left(D \mid G_{1}, G_{\mathrm{b}}\right)$}

As derived by Lorimer et al. (2006), the distribution of pulsars in the Galaxy is not homogeneous, but rather follows a distribution of the form

$$
\rho(R, \psi, z)=\frac{N}{V} \propto R^{B} \exp \left[-\frac{|z|}{E}-C \frac{R-R_{0}}{R_{0}}\right] \mathrm{kpc}^{-3},
$$

with $N$ being the number of pulsars per volume $V$ and constants $R_{0}=8.5 \mathrm{kpc}, B=1.9, C=5$, and $E=330 \mathrm{pc}$ for common pulsars and $E=500 \mathrm{pc}$ for millisecond pulsars (constants from model fit $\mathrm{C}$ and Equations (10) and (11) from Lorimer et al. 2006).

Since the volume density is invariant with the coordinate system used, we can use an Earth-based coordinate system based on the Galactic coordinates of the pulsar and its distance to the Earth, $\left(D, G_{\mathrm{b}}, G_{1}\right)$, for which $\rho\left(D, G_{\mathrm{b}}, G_{1}\right)=\rho(R, \psi, z)$. For the Earth-based observer, the infinitesimal sample volume now becomes

$$
\delta V=D^{2} \delta D \delta \Omega
$$

for a pulsar at given distance $D$ and an infinitesimal solid angle $\delta \Omega$. The number of pulsars in this volume is, hence,

$$
\delta N=\rho\left(D, G_{\mathrm{b}}, G_{1}\right) D^{2} \delta D \delta \Omega .
$$

Since the infinitesimal probability $\delta P$ scales with $\delta N$, we get

$$
p\left(D \mid G_{\mathrm{b}}, G_{1}\right) \propto \rho\left(D, G_{\mathrm{b}}, G_{1}\right) D^{2} .
$$

Consequently, we derive

$$
p\left(D \mid G_{\mathrm{b}}, G_{1}\right) \propto R^{1.9} \exp \left[-\frac{|z|}{E}-5 \frac{R-R_{0}}{R_{0}}\right] D^{2}
$$

with

$$
z\left(D, G_{\mathrm{b}}\right)=D \sin G_{\mathrm{b}}
$$

and

$$
R\left(D, G_{\mathrm{b}}, G_{1}\right)=\sqrt{R_{0}^{2}+\left(D \cos G_{\mathrm{b}}\right)^{2}-2 R_{0} D \cos G_{\mathrm{b}} \cos G_{1}} .
$$




\subsection{The Pulsar Luminosity Term, $p(D \mid S)$}

Finally, since the radio flux, $S$, of pulsars is related to the luminosity, ${ }^{5} L$, of the pulsar through $S D^{2}=L$, this measure can be used to constrain the pulsar distance, through the luminosity distribution of radio pulsars derived by FaucherGiguère \& Kaspi (2006). Considering pulsar luminosities at $1.4 \mathrm{GHz}$ observing frequency with luminosity expressed in units of $\mathrm{mJy} \mathrm{kpc}^{2}$, they proposed a log-normal function with mean $\langle\lambda\rangle=\langle\log (L)\rangle=-1.1$ and standard deviation $\sigma_{\lambda}=0.9$ :

$$
p(\lambda) \propto \exp \left[-\frac{1}{2}\left(\frac{\lambda+1.1}{0.9}\right)^{2}\right]
$$

With $\lambda=\log L=\log S+2 \log D$, we get

$$
p(D) \propto\left|\frac{\partial \lambda}{\partial D}\right| p(\lambda) \propto \frac{1}{D} \exp \left[-\frac{1}{2}\left(\frac{\lambda+1.1}{0.9}\right)^{2}\right],
$$

or, given $S$,

$$
p(D \mid S) \propto \frac{1}{D} \exp \left[-\frac{1}{2}\left(\frac{\log S+2 \log D+1.1}{0.9}\right)^{2}\right] .
$$

Note that this probability is based on the measured radio flux $S$ of the pulsar, not on the $\mathrm{H}$ I flux or the luminosity of an associated SNR or the like. Also, given the analysis of Faucher-Giguère \& Kaspi (2006) who derived the luminosity distribution that we use, the above analysis does not hold for non-radio or bursting pulsars.

\subsection{Combined Distance Probability}

Combining Equations (3), (11), (16), (21), and (26) into Equation (1), we obtain the complete formula for the pulsar distance given the five measurements listed at the start of this section:

$$
\begin{aligned}
& p\left(D \mid \varpi_{\text {meas }}, D_{\text {low }}, D_{\text {up }}, S, G_{1}, G_{\mathrm{b}}\right) \\
& \propto \frac{1}{D^{2}} \exp \left[-\frac{1}{2}\left(\frac{\varpi_{\text {meas }}-1 / D}{\sigma_{\varpi}}\right)^{2}\right] \\
& \quad \times \frac{1}{2}\left[\operatorname{erf}\left(\frac{D_{\text {low }}}{\sqrt{2} \sigma_{\text {low }}}\right)-\operatorname{erf}\left(\frac{D_{\text {low }}-D}{\sqrt{2} \sigma_{\text {low }}}\right)\right] \\
& \quad \times \frac{1}{2}\left[1+\operatorname{erf}\left(\frac{D_{\text {up }}-D}{\sqrt{2} \sigma_{\text {up }}}\right)\right] \\
& \quad \times R^{1.9} D^{2} \exp \left[-\frac{\left|D \sin G_{\mathrm{b}}\right|}{E}-5 \frac{R-R_{0}}{R_{0}}\right] \\
& \quad \times \frac{1}{D} \exp \left[-\frac{1}{2}\left(\frac{\log S+2 \log D+1.1}{0.9}\right)^{2}\right],
\end{aligned}
$$

with $R$ as in Equation (23). Note that the parallax term should be replaced by Equation (6) in case of asymmetric uncertainties. (Technically, Equation (6) can be applied generally to both asymmetric and symmetric cases, but for reasons of clarity, we

\footnotetext{
5 Note that Faucher-Giguère \& Kaspi (2006) define a "pseudo-luminosity" $L=S D^{2}$ that avoids the complexities of emission beam and viewing geometries. This approach is practical for our purposes, and hence we copy their usage of $L$ as an effective "pseudo-luminosity."
}

present the more common, simplified formula here.) Note also that in case measurements are not available, the relevant terms should be omitted, as $p\left(D \mid \varpi_{\text {meas }}\right)$ (for example) is nonsensical in the absence of a $\varpi_{\text {meas }}$ measurement.

Equivalently, we find for the pulsar's parallax

$$
\begin{aligned}
p\left(\varpi \mid \varpi_{\text {meas }}, D_{\text {low }}, D_{\text {up }}, S, G_{1}, G_{\mathrm{b}}\right) \\
\propto\left|\frac{\partial D}{\partial \varpi}\right|^{5} p\left(D \mid \varpi_{\text {meas }}, D_{\text {low }}, D_{\text {up }}, S, G_{1}, G_{\mathrm{b}}\right) \\
\propto \exp \left[-\frac{1}{2}\left(\frac{\varpi_{\text {meas }}-\varpi}{\sigma_{\varpi}}\right)^{2}\right] \\
\quad \times \frac{1}{2 \varpi^{2}}\left[\operatorname{erf}\left(\frac{D_{\text {low }}}{\sqrt{2} \sigma_{\text {low }}}\right)-\operatorname{erf}\left(\frac{D_{\text {low }}-1 / \varpi}{\sqrt{2} \sigma_{\text {low }}}\right)\right] \\
\quad \times \frac{1}{2 \varpi^{2}}\left[1+\operatorname{erf}\left(\frac{D_{\text {up }}-1 / \varpi}{\sqrt{2} \sigma_{\text {up }}}\right)\right] \\
\quad \times \frac{R^{1.9}}{\varpi^{4}} \exp \left[-\frac{\left|\sin G_{\mathrm{b}}\right|}{\varpi E}-5 \frac{R-R_{0}}{R_{0}}\right] \\
\quad \times \frac{1}{\varpi} \exp \left[-\frac{1}{2}\left(\frac{\log S-2 \log \varpi+1.1}{0.9}\right)^{2}\right],
\end{aligned}
$$

where each term contributes a factor $\varpi^{-2}$, since $p\left(\varpi \mid \varpi_{\text {meas }}\right)$ $\delta \varpi=p\left(D \mid \varpi_{\text {meas }}\right) \delta D, p\left(\varpi \mid D_{\text {low }}\right) \delta \varpi=p\left(D \mid D_{\text {low }}\right) \delta D$, etc., implying that each of the five terms contributes a $\delta D / \delta \varpi$ term.

Equation (27) presents the analytic result to the question first discussed by VLM10. However, because in that previous paper parts of the analysis were performed by Monte Carlo simulation, our present results are more accurate, and in contrast to the analysis by VLM10, which only considered parallax, we now derive the full formulae for both distance and parallax. We therefore present in Table 2 the bias-corrected parallax and distance values for the pulsars with parallax measurements first collated by VLM10. Results for pulsars with $\mathrm{H}$ I distance limits (which were not included in VLM10) are presented in Table 1. For the data in these tables, we use the following definitions: the corrected distance $\left(D_{\text {Corr }}\right)$ is the distance for which Equation (27) reaches a maximum; for corrected parallax ( $\varpi_{\text {Corr }}$ ) the same convention is used, based on Equation (28). The $1 \sigma$ uncertainty intervals are defined (consistent with Chatterjee et al. 2009) as the narrowest interval that contains $68 \%$ of the integrated probability density. In practice, this means that a level $P^{*}$ is found so that the integral of $p(D)$ for those values of $D$ where $p(D)>P^{*}$ contains $68 \%$ of the total probability. For bimodal distributions (which only occur toward the Galactic center, and particularly for the measurements for PSR J1752-2806), this may result in two separate regions (a global optimum and a secondary optimum) which in combination contain $68 \%$ probability. Estimation of these quantities is analytically unfeasible and is therefore performed numerically. The code used to calculate the bias-corrected parallax and distance values and uncertainties listed in the tables is available as a supplement to this paper and through an online interface on http://psrpop.phys.wvu.edu/LKbias. An example of the graphical output, showing all five probability terms for PSR J1939+2134 (B1937+21), is shown in Figure 1.

\section{DISCUSSION AND CONCLUSIONS}

Of the 80 pulsars with $\mathrm{H}$ I distance limits, all but one have post-correction distances consistent (at the $1 \sigma$ level assuming the uncertainties derived from our analysis) with the H I limits 

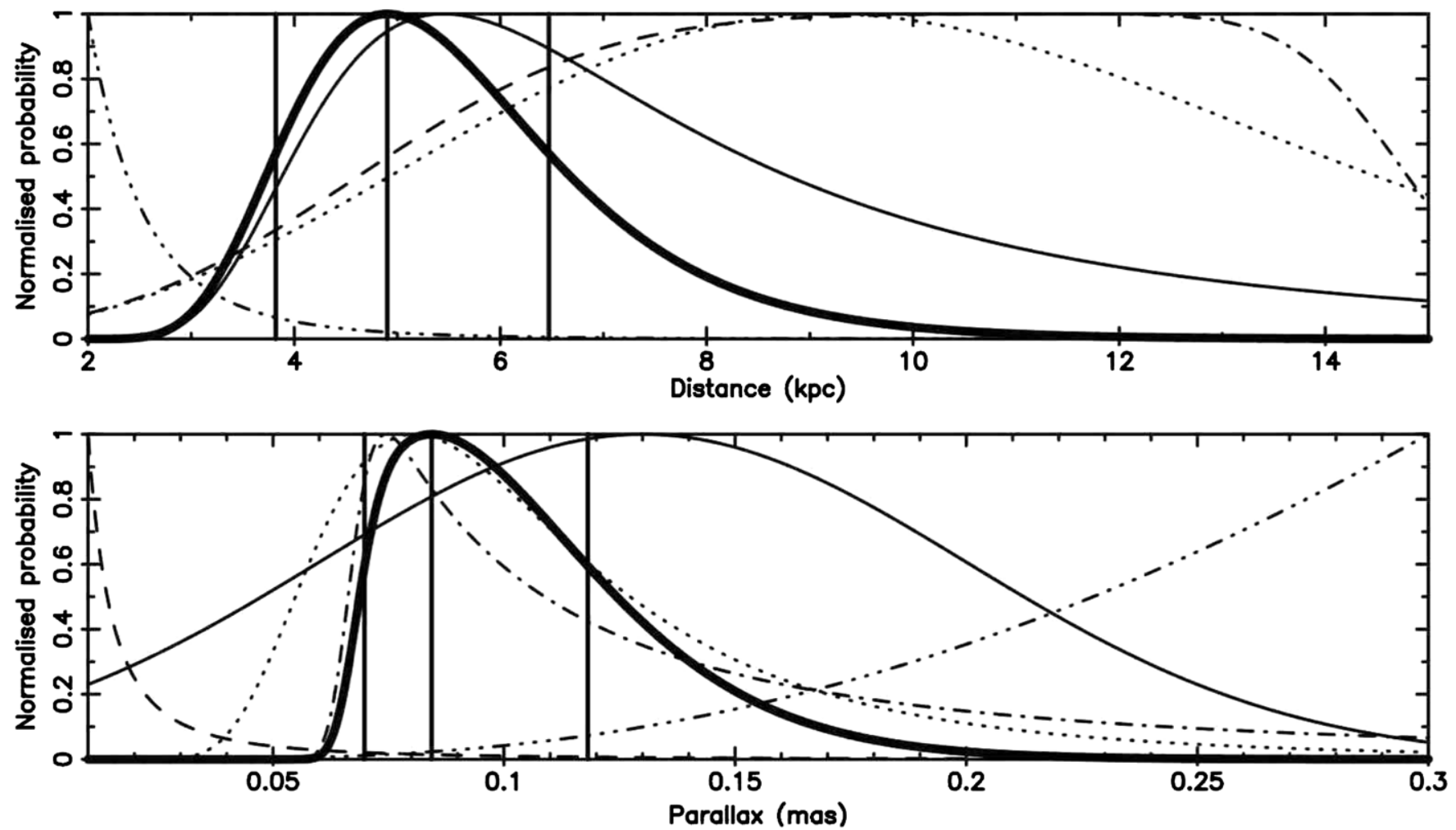

Figure 1. Example output from our likelihood analysis. For PSR J1939+2134, we show the peak-normalized probability distributions of the volumetric (dotted) and luminosity (triple-dot-dashed) terms, as well as the distance limits from $\mathrm{H}$ I estimates (dashed and dot-dashed), the parallax measurement published by Verbiest et al. (2009, thin full line) and the final probability distribution for the pulsar distance (thick full line), with peak and $1 \sigma$ uncertainty interval indicated by the vertical lines. The top figure shows these distributions as a function of distance, while the bottom figure shows the same distributions as a function of parallax. Note that because of the nonlinear relationship between parallax and distance, the most likely distance is not necessarily equal to the inverse of the most likely parallax, although these values do converge for small uncertainties.

published. The exception is PSR J2018+2839 (PSR B2016+28), which has a lower distance limit of $3.2 \pm 2.1 \mathrm{kpc}$, but a parallax measurement of $1.03 \pm 0.10$ mas (Brisken et al. 2002), which dominates the result and therefore makes the $\mathrm{H} \mathrm{I}$ distance limit irrelevant. Furthermore, there is a single source that is beyond the upper $\mathrm{HI}$ distance limit (though within $1 \sigma$ ): this is XTE J1810-197, for which we determine a bias-corrected distance of $3.7 \pm 0.5 \mathrm{kpc}$, which is just beyond the upper distance limit of $3.4 \pm 0.6 \mathrm{kpc}$ derived from H I observations. Since for this neutron star both the lower and the upper limit are equal, and because no radio luminosity is available, the volumetric term determines the slightly higher distance. For 20 sources, the bias-corrected distance is closer than the lower $\mathrm{H}$ I distance limit (though within $1 \sigma$ ) and 59 (or three out of four) sources are completely within the distance limits, with typically biascorrected distances close to the lower $\mathrm{H}$ I distance limit. The fact that our analysis finds that sources are more likely to be closer to the lower rather than upper $\mathrm{H}$ I distance limit (or, indeed, closer even than the lower limit) is unexpected when seen from the perspective presented by Lutz \& Kelker (1973). There are two reasons for this.

First, the upper $\mathrm{H}$ I distance limits are mostly past the tangent point. This means that the volumetric term peaks within-or close to - the range allowed by the $\mathrm{H}$ I limits, which causes the volumetric bias to be either very weak or non-existent. Second, the pulsars to which H I distance limits have been measured, are mostly bright sources, with the exception of the flaring neutron stars and those neutron stars that have $\mathrm{H}$ I limits derived from associations with SNRs. The brightness of these pulsars implies a luminosity term that peaks at very small distances.
Comparing the results in Table 1 and the discussion above with the results in Table 2, it is clear that the types of neutron star distance estimates (parallax and H I measurements) suffer from different statistical biases, although the magnitude of the biases is limited in both cases. While parallax measurements are typically biased toward smaller distances (i.e., the sources are actually further away than suggested by the measurement) because of the relatively limited distance to which this technique works (and the consequential strong effect of the volumetric term), the $\mathrm{H}$ I measurements are typically biased toward larger distances (i.e., the sources are often closer than suggested by the measurement) because the volumetric term has little impact and the luminosity term dominates the analysis.

Finally, of the eight pulsars with both $\mathrm{H}$ I distance limits and parallax distances, only PSR J1857+0943 (B1855+09) has a bias-corrected parallax that is inconsistent with the parallax measurement. The published value of $1.1 \pm 0.2$ mas (Verbiest et al. 2009) is found to be considerably larger than the most likely value of $0.6_{-0.1}^{+0.2}$ mas, which is partly because of the volumetric information (as already found by VLM10, who derived a value of $0.9 \pm 0.2$ mas), but also because of the $\mathrm{H}$ I limits, which place the pulsar well beyond $1 \mathrm{kpc}$.

J.P.W.V. is supported by the European Union under Marie Curie Intra-European Fellowship 236394. J.M.W. and A.A.C. are supported by NSF grant 0807556 . K.L. is supported by ERC Advanced grant "LEAP" (Grant Agreement Number 227947, PI: Kramer). D.R.L. is supported by the West Virginia EPSCoR program and the Research Corporation for Scientific Advancement. We gratefully acknowledge use of the ATNF 
Pulsar Catalogue ${ }^{6}$ for the determination of basic pulsar parameters. The authors thank Lucas Guillemot for useful comments on the draft and Peter den Hartog for interesting discussions.

\section{REFERENCES}

Backer, D. C., \& Fisher, J. R. 1974, ApJ, 189, 137

Bailes, M., Manchester, R. N., Kesteven, M. J., Norris, R. P., \& Reynolds, J. E. 1990, Nature, 343, 240

Brisken, W. F., Benson, J. M., Goss, W. M., \& Thorsett, S. E. 2002, ApJ, 571,906

Brisken, W. F., Thorsett, S. E., Golden, A., \& Goss, W. M. 2003, ApJ, 593, L89

Burgay, M., Joshi, B. C., D’Amico, N., et al. 2006, MNRAS, 368, 283

Chatterjee, S., Brisken, W. F., Vlemmings, W. H. T., et al. 2009, ApJ, 698, 250

Chatterjee, S., Cordes, J. M., Lazio, T. J. W., et al. 2001, ApJ, 550, 287

Chatterjee, S., Cordes, J. M., Vlemmings, W. H. T., et al. 2004, ApJ, 604, 339

Damour, T., \& Taylor, J. H. 1991, ApJ, 366, 501

Deller, A. T., Bailes, M., \& Tingay, S. J. 2009a, Science, 323, 1327

Deller, A. T., Tingay, S. J., Bailes, M., \& Reynolds, J. E. 2009b, ApJ, 701, 1243

Deller, A. T., Tingay, S. J., \& Brisken, W. 2009c, ApJ, 690, 198

Deller, A. T., Verbiest, J. P. W., Tingay, S. J., \& Bailes, M. 2008, ApJ, 685, L67

Dickey, J. M., \& Lockman, F. J. 1990, ARA\&A, 28, 215

Dodson, R., Legge, D., Reynolds, J. E., \& McCulloch, P. M. 2003, ApJ, 596, 1137

Faherty, J., Walter, F. M., \& Anderson, J. 2007, Ap\&SS, 308, 225

Faucher-Giguère, C. A., \& Kaspi, V. M. 2006, ApJ, 643, 332

Fich, M., Blitz, L., \& Stark, A. A. 1989, ApJ, 342, 272

Fomalont, E. B., Goss, W. M., Beasley, A. J., \& Chatterjee, S. 1999, AJ, 117,3025

Frail, D. A., Kulkarni, S. R., \& Vasisht, G. 1993, Nature, 365, 136

Frail, D. A., \& Weisberg, J. M. 1990, AJ, 100, 743

Gaensler, B. M., Brazier, K. T. S., Manchester, R. N., Johnston, S., \& Green, A. J. 1999, MNRAS, 305, 724

Gaensler, B. M., \& Wallace, B. J. 2003, ApJ, 594, 326

Gwinn, C. R., Taylor, J. H., Weisberg, J. M., \& Rawley, L. A. 1986, AJ, 91, 338

Hotan, A. W., Bailes, M., \& Ord, S. M. 2006, MNRAS, 369, 1502

Jacoby, B. A., Bailes, M., Ord, S. M., Knight, H. S., \& Hotan, A. W. 2007, ApJ, 656,408

Jacoby, B. A., Bailes, M., van Kerkwijk, M. H., et al. 2003, ApJ, 599, L99
Johnston, S., Koribalski, B., Weisberg, J. M., \& Wilson, W. 1996, MNRAS 279,661

Johnston, S., Koribalski, B., Weisberg, J. M., \& Wilson, W. 2001, MNRAS, 322,715

Joncas, G., Roger, R. S., \& Dewdney, P. E. 1989, A\&A, 219, 303

Kaplan, D. L., van Kerkwijk, M. H., \& Anderson, J. 2007, ApJ, 660, 1428

Kerr, F. J., \& Lynden-Bell, D. 1986, MNRAS, 221, 1023

Koribalski, B. S., Johnston, S., Weisberg, J., \& Wilson, W. 1995, ApJ, 441, 756

Lazaridis, K., Wex, N., Jessner, A., et al. 2009, MNRAS, 400, 805

Leahy, D. A., \& Tian, W. W. 2008, A\&A, 480, L25

Leahy, D. A., Tian, W. W., \& Wang, Q. D. 2008, AJ, 136, 1477

Lewandowski, W., Wolszczan, A., Feiler, G., Konacki, M., \& Sołtysiński, T. 2004, ApJ, 600, 905

Lommen, A. N., Kipphorn, R. A., Nice, D. J., et al. 2006, ApJ, 642, 1012

Lommen, A. N., Zepka, A., Backer, D. C., et al. 2000, ApJ, 545, 1007

Lorimer, D. R., Faulkner, A. J., Lyne, A. G., et al. 2006, MNRAS, 372, 777

Lutz, T. E., \& Kelker, D. H. 1973, PASP, 85, 573

Manchester, R. N., Hamilton, P. A., \& McCulloch, P. M. 1980, MNRAS, 192,153

Manchester, R. N., Hobbs, G. B., Teoh, A., \& Hobbs, M. 2005, AJ, 129, 1993

McClure-Griffiths, N. M., \& Gaensler, B. M. 2005, ApJ, 630, L161

Minter, A. H., Camilo, F., Ransom, S. M., Halpern, J. P., \& Zimmerman, N. 2008, ApJ, 676, 1189

Nice, D. J., Splaver, E. M., Stairs, I. H., et al. 2005, ApJ, 634, 1242

Ord, S. M., Bailes, M., \& van Straten, W. 2002, MNRAS, 337, 409

Reid, M. J., Menten, K. M., Zheng, X. W., et al. 2009, ApJ, 700, 137

Saravanan, T. P., Deshpande, A. A., Wilson, W., et al. 1996, MNRAS, 280, 1027

Stairs, I. H., Thorsett, S. E., Taylor, J. H., \& Wolszczan, A. 2002, ApJ, 581, 501

Strom, R. G., \& Stappers, B. W. 2000, in ASP Conf. Series, vol. 202, Pulsar Astronomy-2000 and Beyond, ed. M. Kramer, N. Wex, \& R. Wielebinski (San Francisco, CA: ASP), 509

Tian, W. W., \& Leahy, D. A. 2008a, ApJ, 677, 292

Tian, W. W., \& Leahy, D. A. 2008b, MNRAS, 391, L54

Tian, W. W., Leahy, D. A., \& Li, D. 2010, MNRAS, 404, L1

van Kerkwijk, M. H., \& Kaplan, D. L. 2007, Ap\&SS, 308, 191

Verbiest, J. P. W., Bailes, M., Coles, W. A., et al. 2009, MNRAS, 400, 951

Verbiest, J. P. W., Lorimer, D. R., \& McLaughlin, M. A. 2010, MNRAS, 405, 564

Weisberg, J. M., Siegel, M. H., Frail, D. A., \& Johnston, S. 1995, ApJ, 447, 204

Weisberg, J. M., Stanimirović, S., Xilouris, K., et al. 2008, ApJ, 674, 286

Wolszczan, A., Doroshenko, O., Konacki, M., et al. 2000, ApJ, 528, 907

\footnotetext{
6 Manchester et al. (2005); current online version at
} http://www.atnf.csiro.au/research/pulsar/psrcat/ 